\title{
Avaliação in vitro da acurácia de um novo localizador eletrônico foraminal
}

\author{
In vitro evaluation of the accuracy of a new \\ foraminal electronic locator
}

\section{Isaac de Sousa Araújo' 1 (1) Anamim Larissa Querendo de Oliveira² (1) Cirdes Ferreira Borges 3 (D)}

${ }^{1}$ Autor para correspondência. Centro Universitário Doutor Leão Sampaio. Juazeiro do Norte, Ceará, Brasil. isaacaraujo@leaosampaio.edu.br
2,3Centro Universitário Doutor Leão Sampaio. Juazeiro do Norte, Ceará, Brasil. analaryy@hotmail.com, cirdes@hotmail.com

RESUMO |OBJETIVO: Avaliar, em condição in vitro, a acurácia das leituras odontométricas obtidas por meio do localizador eletrônico foraminal iRoot Apex. MÉTODOS E MATERIAIS: trinta e um pré-molares inferiores humanos uniradiculados tiveram seus acessos, ampliação cervical e aferição do comprimento real realizados de forma padronizada. As medições eletrônicas foram realizadas nos pontos 1.0, 0.5 e 0.0, marcados na tela do aparelho, e comparadas as medidas reais a $1 \mathrm{~mm}, 0,5 \mathrm{~mm}$ e no forame apical, respectivamente. Os dados foram tabulados e analisados utilizando-se os testes ANOVA e Bonferroni, com significância de 5\%. RESULTADOS: Os resultados foram calculados em função dos valores absolutos dos erros médios e da acurácia $( \pm 0,5 \mathrm{~mm})$ em cada referência de medição, obtendo-se respectivamente: $0,19 \mathrm{~mm}$ e $96,8 \%$ (0.0), 0,21 mm e $96,8 \%$ (0.5), 0,34 mm e 74,1\% (1.0). Diferenças significantes foram observadas apenas entre os pontos mais apicais (0.0 e 0.5) e as aferições realizadas na marcação 1.0 ( $P$ $<0,05)$. CONCLUSÃo: nas condições deste estudo, o aparelho iRoot Apex® apresentou alta acurácia, todavia, as medições eletrônicas realizadas no ponto 1.0 do aparelho apresentaram acurácia inferior às medidas eletrônicas obtidas no forame ou próximo deste ponto anatômico.

PALAVRAS-CHAVE: Endodontia. Odontometria. Ápice dentário.
ABSTRACT |OBJECTIVE: To evaluate, in vitro condition, the accuracy of the electronic readings obtained through the foraminal electronic locator iRoot Apex. METHODS AND MATERIALS: Thirty-one uniradicular human mandibular premolars had their accesses, cervical enlargement and real length measurement performed in a standardized way. Electronic measurements were performed at points 1.0, 0.5 and 0.0 marked on the device's dispaly and compared to the actual measurements at $1 \mathrm{~mm}, 0.5 \mathrm{~mm}$ and at the apical foramen, respectively. The data were tabulated and analyzed using the ANOVA and Bonferroni tests with a $5 \%$ significance. RESULTS: The results were calculated as a function of the absolute values of the average errors and the accuracy $( \pm 0.5$ $\mathrm{mm}$ ) in each measurement reference, obtaining respectively: $0.19 \mathrm{~mm}$ and $96.8 \%(0.0), 0,21 \mathrm{~mm}$ and $96.8 \%(0.5), 0.34$ $\mathrm{mm}$ and $74.1 \%$ (1.0). Significant differences were observed only between the most apical points $(0.0$ and 0.5$)$ and the measurements made in marking $1.0(P<0.05)$. CONCLUSION: in the conditions of this study, the iRoot Apex® device showed high accuracy, however, the electronic measurements performed in point 1.0 of the device are less accurate than the electronic measurements obtained in the foramen or close to this anatomical point.

KEYWORDS: Endodontics. Odontometry. Tooth apex. 


\section{Introdução}

A correta determinação do limite apical de trabalho é um dos fatores determinantes para o sucesso do tratamento endodôntico ${ }^{1,2}$. Existem alguns métodos utilizados para se chegar ao comprimento de trabalho, sendo que o mais empregado atualmente ainda é o radiográfico, por meio de periapicais, no momento da odontometria. Porém esse recurso apresenta limitações, uma vez que a radiografia periapical pode apresentar muitas distorções, não sendo tão fidedigna para o propósito odontométrico 3,4 .

Na tentativa de mitigar erros nas etapas operatórias, desenvolveram-se tecnologias que buscam aumentar a taxa de sucesso da terapia endodôntica, tornandoa mais previsível ${ }^{5}$. Assim, os estudos sobre odontometria eletrônica culminaram na criação e desenvolvimento dos Localizadores Eletrônicos Foraminais (LEFs), que passaram por uma grande evolução desde os achados de Custer ${ }^{-6}$, com os primeiros equipamentos que funcionavam baseados na resistência elétrica dos tecidos e, portanto, eram bastante imprecisos sob a presença de tecido pulpar vital, excesso de sangue ou umidade no interior do canal radicular, até os mais modernos, que funcionam por meio de uma corrente elétrica alternada em duas ou mais frequências e determinam, por meio de diferentes métodos, a posição dos instrumentos endodônticos no interior dos canais radiculares de forma independente ou dinâmica, acoplados ao motor ${ }^{?}$.

Os resultados dos estudos atuais, utilizando diferentes metodologias, mostram que os LEFs são acurados dentro de um limite clinicamente aceitável de \pm $0,5 \mathrm{~mm}$, têm mais aceitação pelo paciente e maior facilidade de uso pelos cirurgiões-dentistas, se configurando como ferramenta indispensável para a clínica endodôntica diária $4,8,9$.

O iRoot Apex® (BASSI $^{\mathrm{TM}}$, Brasil) é um aparelho introduzido recentemente no mercado e o seu sistema de leitura utiliza uma tela de LCD (Liquid Crystal Display) de tamanho reduzido, alta resolução e com possibilidade de observação dos parâmetros de leitura em diferentes ângulos. O aparelho possui ainda possibilidade de transmissão de sinal via bluetooth, ajuste do limite apical pelo operador e funcionamento com bateria interna ${ }^{10}$.

Com a consolidação dos LEFs como método e ferramenta de extrema importância na determinação do limite apical de trabalho, novos modelos destes aparelhos estão disponíveis com pouca ou nenhuma investigação científica, como é o caso do iRoot Apex®, devendo então serem verificados sua precisão e confiabilidade. $\mathrm{O}$ objetivo do presente trabalho foi verificar, in vitro a acurácia das leituras eletrônicas obtidas por meio do localizador eletrônico foraminal iRoot Apex ${ }^{\circledR}$ nas referências $0.0,0.5$ e 1.0 da tela do apareIho, comparando com comprimentos reais obtidos no forame apical e à $0,5 \mathrm{~mm}$ e $1,0 \mathrm{~mm}$ aquém deste.

\section{Métodos e materiais}

\section{Preparo dos espécimes}

A coleta, preparo dos espécimes e os ensaios metodológicos in vitro foram realizados nas dependências do Centro Universitário Doutor Leão Sampaio, mediante parecer prévio do Comitê de Ética em Pesquisa vinculado à instituição (parecer $n^{\circ}$ 3.017.361).

Trinta e um pré-molares humanos inferiores unirradiculados, com ápices completamente formados e íntegros, sem curvatura radicular acentuada $\left(>25^{\circ}\right)$, extraídos por razões protéticas, ortodônticas e/ou periodontais foram selecionados para este estudo. Foram excluídos dentes com canais múltiplos, reabsorções radiculares comunicantes, calcificação pulpar, com algum tipo de fator limitante à patência radicular ou forames apicais patentes à limas K > \#20.

A amostra, após a sua coleta, foi imersa em solução de hipoclorito de sódio a 2,5\% (Biodinâmica, Brasil) por duas horas. Restos teciduais, cálculos e outras sujidades foram removidos com auxílio de curetas periodontais e um aparelho de ultrassom. Em seguida os dentes foram lavados em água corrente, esterilizados e conservados em solução de timol a 0,01\%.

Os acessos coronários foram realizados de maneira padronizada utilizando pontas diamantadas $n^{\circ} 1012$ e 3081 (KG Sorensen, Brasil) acionadas em alta rotação sob abundante irrigação. Superfícies planas na porção oclusal dos dentes foram criadas com o objetivo de servirem como ponto seguro para o posicionamento dos limitadores de penetração dos instrumentos endodônticos.

A exploração inicial dos canais radiculares foi executada com limas manuais tipo K\#10, 31 mm (Dentsply/ 
Maillefer, Suíça) e restos de tecido pulpar, quando presentes, foram removidos cuidadosamente, no intuito de eliminar interferências intracanais que pudessem afetar o ajuste dos instrumentos. A partir de então, os dentes foram numerados e o Comprimento Real do Canal (CRC) de cada espécime foi determinado por meio do método direto, realizando-se a introdução de lima manual tipo $\mathrm{K} \# 15$, até a visualização de suas pontas ao nível foraminal com o auxílio de mini microscópio digital (CE FC Rohs, China) com magnificação 20X. Depois de ajustar o limitador de penetração na referência oclusal de cada dente, a distância entre a ponta da lima e a margem inferior do limitador foi aferida com auxílio de paquímetro digital com precisão de $\pm 0,01 \mathrm{~mm}$ (MTX, China). A partir da medida de CRC, foram subtraídos $0,5 \mathrm{~mm}$ e 1,0 mm para estabelecimento das outras medidas de referência para futuras comparações. Em caso de eliminação de algum dos espécimes outros foram preparados para substituí-los.

Seguindo os princípios biomecânicos e recomendações do fabricante, para realização da odontometria eletrônica, foi efetuado o pré-alargamento dos terços cervical e médio, por meio do emprego de brocas Gates-Glidden \# 3 e \#2(Dentsply/Maillefer, Suíça) acionadas em baixa rotação, até dois terços do comprimento real do dente. $O$ canal foi irrigado com 1,0 $\mathrm{ml}$ de $\mathrm{NaOCl}$ a 2,5\% a cada troca de broca por meio de seringa endodôntica com agulha hipodérmica 20 $x$ 0,55 mm. Concluída esta fase, os espécimes tiveram seus forames padronizados por meio de instrumentação manual até um instrumento tipo $\mathrm{K}$ com diâmetro de ponta de $250 \mu \mathrm{m}$, inserido até o CRC.

\section{Obtenção das medidas eletrônicas}

Após o preparo inicial, cada espécime foi montado em um modelo experimental composto por um recipiente plástico de polietileno com dois furos na tampa, um central, para fixação do dente, e outro próximo ao bordo, para colocação do clipe labial do LEF. Para melhorar a estabilização do dente, sobre o furo central da tampa foi colado um anel de borracha com adesivo instantâneo à base de cianocrilato. Para haver a condução elétrica o recipiente foi preenchido com solução salina a 0,9\% (Eurofarma, Brasil).

Todas as medidas eletrônicas foram obtidas por um único operador, previamente calibrado e sem conhecimento do CRC, utilizando o LEF iRoot Apex (Fig. 1), com carga máxima de energia, de acordo com as instruções do fabricante. Os canais radiculares foram preenchidos com hipoclorito de sódio e em seguida um instrumento manual tipo $\mathrm{K} \# 25,31 \mathrm{~mm}$, que foi inserido exercendo ligeira pressão apical até que o visor do aparelho mostrasse a indicação 0.0 (APEX) no display do LEF, considerando-se válida a leitura que permaneceu estável por pelo menos cinco segundos. A posição do cursor de borracha foi ajustada até a referência oclusal, o instrumento foi desconectado do LEF, removido do interior do canal, a distância entre a ponta do instrumento e o cursor de borracha foi mensurado com um paquímetro digital e anotada como Comprimento Eletrônico do Canal (CEC). As leituras nas posições 0.5 e 1.0, indicadas no display do LEF, seguiram esse mesmo protocolo, com o uso de limas de tipo $\mathrm{K} \# 30$ e \#35, que melhor se ajustaram aos respectivos limites.

Figura 1. Momento da medição eletrônica com o aparelho iRoot Apex®

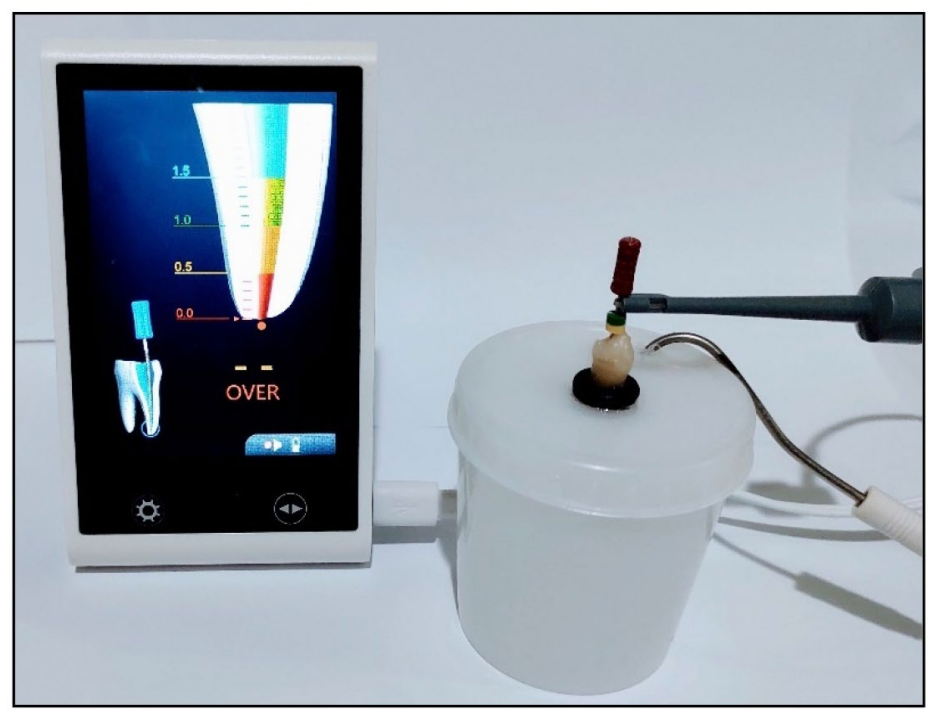

. Dent. Public. Health, Salvador, 2020 Dezembro;11(2):103-110 http://dx.doi.org/10.17267/2596-3368dentistry.v11v2.3097 | ISSN: 2596-3368 


\section{Análise estatística}

A análise estatística dos resultados do estudo foi realizada em função dos erros médios do dispositivo, aferidos em milímetros, nas três posições do $\operatorname{LEF}(0.0,0.5$ e 1.0) até o CRC, CRC-0,5 mm e CRC-1,0 mm, respectivamente. A análise descritiva dos resultados observou o padrão das determinações, se aquém ou além do desejado, a ocorrência de medidas além do forame apical e a frequência de aferições em nível aceitável, considerando margem de erro estabelecida em $\pm 0,5 \mathrm{~mm}$. Os valores de erro médio foram levados à curva de normalidade onde foi determinada a natureza paramétrica dos dados pelo teste de Shapiro-Wilk. A análise estatística foi conduzida utilizando o teste ANOVA para as comparações entre as aferições do LEF nos três níveis de medição, e o teste de Bonferroni para verificar as diferenças entre cada nível de medição. Para ambos os testes foi estabelecido um nível de significância de 5\%, usando o software SPSS v.20 (SPSS Inc., EUA).

\section{Resultados}

A distribuição das diferenças obtidas a partir da medição do comprimento real do canal, pela visualização direta da ponta da lima pelo forame apical, e da medição eletrônica utilizando o localizador eletrônico foraminal iRoot Apex®, nos três níveis, é mostrada na tabela 1.

Tabela 1. Diferenças entre as medidas de CRC e CEC para as medições realizadas em 0.0, 0.5 e 1.0 do display do LEF

\begin{tabular}{|c|c|c|c|c|c|c|c|c|}
\hline \multirow{2}{*}{$\begin{array}{c}\text { Diferença entre o CEC e o CRC } \\
(\mathrm{mm})\end{array}$} & \multicolumn{2}{|c|}{0.0} & \multicolumn{2}{|c|}{0.5} & \multicolumn{2}{|c|}{1.0} & \multicolumn{2}{|c|}{ Total } \\
\hline & $N$ & $\%$ & $n$ & $\%$ & $n$ & $\%$ & $n$ & $\%$ \\
\hline$<-0,51^{*}$ & 01 & 3,2 & 01 & 3,2 & 03 & 9,7 & 05 & 5,4 \\
\hline$-0,50$ a $-0,01 *$ & 18 & 58,1 & 16 & 51,7 & 13 & 41,9 & 47 & 50,5 \\
\hline 0,00 & 03 & 9,7 & 01 & 3,2 & 01 & 3,2 & 05 & 5,4 \\
\hline 0,01 a 0,50 & 09 & 29,0 & 13 & 41,9 & 09 & 29,0 & 31 & 33,3 \\
\hline$>0,51$ & 00 & 0,0 & 00 & 0,0 & 05 & 16,2 & 05 & 5,4 \\
\hline
\end{tabular}

Legenda: * Valor negativo indica a posição da lima aquém (posição coronal) do CRC.

As taxas de precisão do aparelho testado foram $96,8 \%, 96,8, \%$, e $74,1 \%$ para os níveis $0.0,0.5$ e 1.0, respectivamente, com tolerância definida em $\pm 0,5 \mathrm{~mm}$. Em nenhuma das medições eletrônicas realizadas com o iRoot Apex ${ }^{\circledR}$ foram observadas medidas maiores que CRC, descontando-se a margem de tolerância inerente à metodologia.

A tabela 2 apresenta os valores médios e de desvio padrão, obtidos pelo teste de análise de variância ANOVA, dos Erros entre as medidas eletrônicas e o Comprimento Real do Canal até o forame apical, e a 0,5 mm e 1,0 mm aquém deste ponto anatômico, bem como os valores mínimo e máximo encontrados com o LEF em cada nível.

As medidas eletrônicas realizadas com o iRoot Apex® forneceram os menores erros médios no nível 0.0 (APEX), representando os melhores resultados, todavia, não foram encontrados valores estatisticamente diferentes entre essas medidas e as aferições realizadas na referência $0.5(p<0,05)$. As leituras executadas no ponto 1.0 do aparelho demonstraram menores valores de precisão que os demais níveis, com erro médio maior e estatisticamente diferente das aferições realizadas nos níveis mais apicais. 
Tabela 2. Erro médio e desvio padrão (DP) da medida eletrônica em relação ao Comprimento Real do Canal (mm) em cada nível de aferição

\begin{tabular}{ccccc}
\hline Nível de Aferição & Média* $^{*}$ & Valor mínimo & Valor máximo & Desvio padrão \\
\hline 0.0 & $0,19^{\mathrm{a}}$ & 0,00 & 0,52 & 0,14 \\
0.5 & $0,21^{\mathrm{a}}$ & 0,00 & 0,64 & 0,14 \\
1.0 & $0,34^{\mathrm{b}}$ & 0,00 & 0,66 & 0,20 \\
\hline
\end{tabular}

Legenda: *Erro médio em termos de valores absolutos das determinações.

Valores numéricos seguidos por letras diferentes indicam diferenças estatisticamente significantes em função dos testes ANOVA e Bonferroni $(P<0,05)$.

\section{Discussão}

A realização desta pesquisa seguiu alguns cuidados com a finalidade de mitigação de vieses metodológicos. A realização do procedimento de ampliação cervical prévio à execução da fase de odontometria foi demonstrado como relevante pela literatura, observando-se aumento da precisão das leituras eletrônicas realizadas após o preparo cervical11,12. Assim como, a ausência de patência foraminal é fator importante para a interpretação adequada por alguns LEFs, interferindo em sua precisão $\stackrel{13}{ }$.

A padronização do forame apical em um diâmetro de 250 um, mesmo diâmetro da ponta das limas utilizadas durante a fase de odontometria, teve como objetivo evitar quaisquer variações na obtenção das leituras eletrônicas. Essa padronização foi apontada como medida importante para o aumento da precisão dos LEFs, visto que o maior ajuste do instrumento à constrição apical melhora significativamente o desempenho destes aparelhos ${ }^{14}$. Esse fato, e sua relacão com a característica cônica do canal radicular, também justifica o emprego de instrumento com calibres maiores nos limites subsequentes, mais cervicais ${ }^{15}$.

O uso do microscópio como auxiliar na obtenção do Comprimento Real do Dente pelo método direto possibilitou a determinação desta medida com maior precisão, visto que sem a utilização dessa ferramenta a aferição ficaria dificultada, principalmente pela margem de erro de $0,5 \mathrm{~mm}$ estabelecida para a metodologia empregada nesta pesquisa. O uso de microscópio para esta etapa metodológica já foi relatado pela literatura ${ }^{16,17}$.

O modelo experimental utilizado nesse estudo foi semelhante ao usado por outros autores, que mostraram que a solução salina 0,9\% é um bom meio condutor para a realização da odontometria em técnica in vitro ${ }^{5,17}$.

O aparelho utilizado, o iRoot Apex®, com processo de medição baseado no método da impedância de multifrequência e calibração automática que, segundo o fabricante, garante medições mais precisas. A interface de aferição possibilitou a observação dinâmica do instrumento no interior do canal através de um diagrama esquemático de cores, gradualmente preenchidas de acordo com as diferentes posições da lima no endodonto. Sinais sonoros de diferentes intensidades também alertaram quando a lima se aproximou ou atingiu os pontos de referência predefinidos ${ }^{10}$.

Apesar das limitações inerentes às investigações experimentais de laboratório, referentes à extrapolação de resultados para seres humanos, devido às dificuldades em reproduzir todas as variáveis potenciais que podem estar presentes em um ambiente clínico ${ }^{18}$, este estudo in vitro figura como uma fonte importante de parâmetros para o uso do LEF iRoot Apex® durante a prática clínica.

No presente estudo, considerando a margem de tolerância de $\pm 0,5 \mathrm{~mm}, \mathrm{o}$ LEF iRoot Apex® apresentou uma alta acurácia $(89,2 \%)$, independente do limite considerado, havendo alta precisão nas aferições obtidas nas posições 0.0 e 0.5 do display, $96,8 \%$ em ambos em contraponto com as aferições realizadas no nível 1.0, que obtiveram os menores valores de acurácia (74,1\%). Tais achados corroboram com os resultados de Vasconcelos et al. ${ }^{19}$ que relataram redução da acurácia dos LEFs Root ZX, RomiApex D-30 e Ipex quando os instrumentos não foram inseridos até o forame apical (para medições no nível de $1,0 \mathrm{~mm}$ ), de $81,6 \%$ para $60,5 \%$, de $65,8 \%$ para $36,8 \%$ e de $55,3 \%$ para $44,7 \%$, respectivamente, considerando $0,5 \mathrm{~mm}$ como margem de tolerância. 
De acordo com as informações obtidas no presente estudo, com relação às medidas de erro entre as aferições eletrônicas e os comprimentos reais, não houveram leituras realizadas além dos limites do forame apical, em acordo com o estudo de Maachar et al. 20 que, avaliando a precisão de leitura do localizador apical eletrônico Novapex, não encontrou medida além do comprimento real do dente, estabelecido no forame. Esses achados são de grande importância e fazem dos LEFs ferramentas únicas na determinação do comprimento de trabalho e justificam as recomendações de vários autores que sugeriram a realização da instrumentação à nível foraminal ou a 0,5 $\mathrm{mm}$ deste (conforme detectado pelo dispositivo) para evitar instrumentação e/ou obturação além dos limites desejados $21-23$.

O presente estudo buscou, ainda, comparar as medidas obtidas nas posições $0.0,0.5$ e 1.0 do display do iRoot Apex ${ }^{\circledR}$ com comprimentos reais obtidos no forame apical, 0,5 mm e 1,0 $\mathrm{mm}$ aquém deste. Os resultados mostraram que a média dos erros entre os respectivos pares de referências aumenta à medida em ocorre o distanciamento do forame em direção coronária, havendo um maior erro médio entre a referência 1.0 e a medida de CRC-1,0 mm, com diferença estatisticamente significante $(p<0,05)$ em relação aos erros médios obtidos nas posições mais apicais. Quando foram analisadas a relação entre os valores obtidos nas referências 0.0 e 0.5 da tela e seus respectivos comprimentos reais, estes apresentaram semelhança estatística. Estas observações sugerem que há uma alta correlação entre os pontos 0.0 e 0.5 anotadas no display do LEF iRoot Apex ${ }^{\circledR}$ e as leituras obtidas no forame e 0,5 mm aquém deste.

A literatura ainda é escassa em relação a estudos com este aparelho, pelo fato do seu lançamento relativamente recente no mercado, porém Higa et al. $\underline{24}$, após avaliar e comparar a relação entre a distância da ponta da lima ao forame apical e areferência mostrada no display de três localizadores apicais eletrônicos, Dentaport ZX® (J. Morita Co., Kyoto, Japan), o Justy $I I \AA$ (Yoshida Co., Tokyo, Japan) e o E-Magic Finder Deluxe ${ }^{\circledR}$ (DESTI S-Denti Co. Ltd, Chungnam, Korea), constataram que a precisão das leituras mostradas no visor dos LEFs foi inversamente proporcional à distância da ponta do instrumento ao forame apical, corroborando com os achados desse estudo, em que a precisão das medições aumenta quando a ponta da lima se aproxima do forame apical.
Embora os resultados deste estudo apontem para uma alta acurácia do LEF iRoot Apex®, principalmente nas marcações 0.0 e 0.5 do display, as condições encontradas em estudos in vitro são comparativamente mais controladas que às encontradas in vivo. Por esse motivo, esses resultados não podem ser aplicados diretamente ao trabalho clínico, embora reflitam uma tendência de precisão deste LEF.

\section{Conclusão}

Dentro das limitações do presente trabalho, pode-se afirmar que o localizador eletrônico foraminal iRoot Apex ${ }^{\circledR}$ demonstrou alta precisão junto ao forame apical e a 0,5 $\mathrm{mm}$ aquém deste. No entanto, quando as medições foram feitas à distância de $1 \mathrm{~mm}$ aquém dessa estrutura anatômica, o dispositivo sofreu diminuição de sua precisão.

Ainda, há uma correlação entre a posição da lima no interior do canal e as marcações 0.0 e 0.5 mostradas no display do localizador eletrônico foraminal iRoot Apex ${ }^{\circledR}$, diferente estatisticamente da posição 1.0.

Faz-se necessário, que futuramente, sejam realizadas pesquisas in vivo para verificar a acurácia deste localizador.

\section{Contribuições dos autores}

Oliveira ALQ foi responsável pela montagem do experimento e coleta. Borges CF foi responsável pela montagem do experimento e coleta. Araújo IS foi responsável pela tabulação, análise estatística dos dados, criação de tabelas e figuras e redação do texto. Todos os autores revisaram e aprovaram a versão final.

\section{Conflitos de interesses}

Nenhum conflito financeiro, legal ou político envolvendo terceiros (governo, empresas e fundações privadas, etc.) foi declarado para nenhum aspecto do trabalho submetido (incluindo, mas não se limitando a subvenções e financiamentos, participação em conselho consultivo, desenho de estudo, preparação de manuscrito, análise estatística, etc.). 


\section{Referências}

1. Basmadjian-Charles $C L$, Farge $P$, Bourgeois DM, Lebrun $T$. Factors influencing the long-term results of endodontic treatment: a review of the literature. Int Dent J. 2002;52(2):81-86. http:// dx.doi.org/10.1111/j.1875-595x.2002.tb00605.x

2. Ng YL, Mann V, Rahbaran S, Lewsey J, Gulabivala K. Outcome of primary rootcanaltreatment: systematic review of the literaturepart 1. Effects of studycharacteristics onprobability of success. Int Endod J. 2007;40(12):921-39. http://dx.doi.org/10.1111/j.13652591.2007.01322.x

3. Bonetti C, Armond MC, Gazolla MS, Corsetti SA, Pereira LJ. Avaliação comparativa entre dois métodos na odontometria: eletrônico e radiográfico. Arq bras odontol. 2010;3(1):17-24.

4. Nóbrega WFS, Dantas AE, Rosendo RA, Sarmento TCAP. Análise comparativa da precisão e da confiabilidade de dois localizadores eletrônicos foraminais: um estudo in vitro. Revista da Faculdade de Odontologia-UPF. 2016;21(1):15-22. http://dx.doi.org/10.5335/ rfo.v21i1.5343

5. Venante HS, Vilar GC, Yamashita F, Dias OHS, Silva Junior ES, Interliche R. Análise da acurácia de dois localizadores apicais eletrônicos: um estudo in vitro. Braz J Surg Clin Res. 2017;19(3):2731.

6. Custer LE. Exact methods of locating the apical foramen. JADA. 1918;5(8):815-819. http://dx.doi.org/10.14219/jada. archive.1918.0368

7. Guimarães BM, Marciano MA, Amoroso-Silva PA, Alcade MP, Bramante CM, Duarte MAH. O uso dos localizadores foraminais na endodontia: revisão de literatura. Rev Odontol Bras Central. 2014;23(64):2-7.

8. Oliveira TN, Vivacqua-Gomes N, Bernardes RA, Vivan RR, Duarte MAH, Vasconcelos BC. Determination of the accuracy of 5 electronic apex locators in the function of different employment protocols. J Endod. 2017;43(10):1663-1667. http://dx.doi. org/10.1016/j.joen.2017.03.039

9. Yolagiden M, Ersahan S, Suyun G, Bilgec E, Aydin C.Comparison of Four Electronic Apex Locators in Detecting Working Length: An Ex Vivo Study. J Contemp Dent Pract. 2018;19(12):1427-1433. http://dx.doi.org/10.5005/jp-journals-10024-2444

10. BASSI. Manual do operador para o iRoot Apex [Internet]. 2019. Disponível em: https://easy.odo.br/wp-content/uploads/2020/07/ Manual_iRootApex-1.pdf
11. Camargo EJ, Zapata RO, Medeiros PL, Bramante CM, Bernardineli N, Garcia RB et al. Influence of preflaring on the accuracy of length determination with four electronic apex locators. J Endod. 2009;35(9):1300-2. http://dx.doi.org/10.1016/j. joen.2009.05.030

12. Anele JA, Tedesco M, Marques-Da-Silva B, Baratto Filho F, Leonardi DP, Harahushiku G et al. Análise ex vivo da influência do preparo cervical na determinação do comprimento de trabalho por três diferentes localizadores apicais eletrônicos. RSBO Revista Sul-Brasileira de Odontologia. 2010;7(2):139-45.

13. Vasconcelos BC, Chaves RDV, Vivacqua-Gomes N, Candeiro GTM, Bernardes RA, Vivan RR et al. Ex vivo evaluation of the accuracy of electronic foramen locators in root canals with an obstructed apical foramen. J Endod. 2015;41(9):1551-4. http:// dx.doi.org/10.1016/j.joen.2015.06.009

14. Vasconcelos BC, Matos LA, Pinheiro-Júnior EC, Menezes AST, Vivacqua-Gomes N. Ex vivo accuracy of three electronic apex locators using different apical file sizes. Braz Dent J. 2012;23(3):199-204. http://dx.doi.org/10.1590/S0103$\underline{64402012000300003}$

15. Baldi JV. Influência do diâmetro do forame apical e do calibre do instrumento endodôntico nas leituras odontométricas proporcionadas por dois aparelhos localizadores apicais [dissertação]. São Paulo: Universidade de São Paulo; 2005.

16. Cruz ATG, Wichnieski C, Carneiro E, Silva Neto UX, Gambarini G, Piasecki L. Accuracyof 2 endodonticrotarymotorswithintegratedapexlocator. J Endod. 2017;43(10):1716-1719. http://dx.doi.org/10.1016/j. joen.2017.05.012

17. Parirokh M, Manochehrifar $H$, Abbott PV, Borna R, Haghdoost AA. Effect of Various Electronic Devices on the Performance of Electronic Apex Locator. Iran Endod J. 2019;14(4):278-82. http:// dx.doi.org/10.22037/iej.v14i4.23752

18. Chita JJ, Silva PG, Pereira KFS, Onoda HK, Borba Junior JC, Ramos CAS. Precisão e confiabilidade de um novo localizador foraminal eletrônico-Estudo in vivo. Pesq Bras Odonto Ped Clin Integr. 2012;12(4):457-63. http://dx.doi.org/10.4034/ PBOCI.2012.124.02

19. Vasconcelos BC, Vale TM, Menezes AST, Pinheiro-Junior EC, Vivacqua-Gomes N, Bernardes RA et al. An ex vivo comparison of root canal length determination by three electronic apex locators at positions short of the apical foramen. Oral Surg Oral Med Oral Pathol Oral Radiol Endod. 2010;110(2):e57-61. http://dx.doi. org/10.1016/j.tripleo.2010.03.005 
20. Maachar DF, Silva PG, Barros RMG, Pereira KFS. Avaliação da precisão do localizador apical Novapex: estudo in vitro. Revista de Odontologia da UNESP. 2008;37(1):41-46.

21. Choudary TM, Deosarkar B, Chavan S, Kurtikar K, Darak P. Comparison of accuracy of root ZX, propexpixi and romidan apex locator-an in-vitro study. Journal of Applied Dental and Medical Sciences. 2017;3(4):6-12.

22. Zand V, Rahimi S, Davoudi P, Afshang A. Accuracy of Working Length Determination using NovApex and Root-ZX Apex Locators: An in vitro Study. J Contemp Dent Pract. 2017;18(5):383-385.

http://dx.doi.org/10.5005/jp-journals-10024-2051
23. Aggarwal G, Bogra P, GuptaS, Jindal A, Jain N. Determination of apical constriction and apical foramen using electronic apex locator in vivo: Comparison between vital and nonvital teeth. Saudi Endodontic Journal. 2018;8(2)99-105. http://dx.doi. org/10.4103/sej.sej_35_17

24. Higa RA, Adorno CG, Ebrahim AK, Suda H. Distance from file tip to the major apical foramen in relation to the numeric meter reading on the display of three different electronic apex locators. Int Endod J. 2009;42(12):1065-70. http://dx.doi.org/10.1111/j.13652591.2009.01629.x 